\title{
Hormonas utilizadas como agentes ergogénicos: situación actual del problema
}

\author{
Hormones used as ergogenics: present state of the question
}

\author{
C. Laudo ${ }^{1}$, V. Puigdevall ${ }^{2}$, M. J. del Río ${ }^{1}$, A. Velasco ${ }^{1}$
}

\section{RESUMEN}

En nuestra sociedad, la competición deportiva se ha convertido en el espectáculo que moviliza y reúne mayor número de personas en todo el mundo, con la correspondiente influencia cultural y económica que de ello deriva. Por lo que, los deseos de superación atlética han llevado a los deportistas a practicar intensos programas de entrenamiento y a recurrir al consumo de sustancias que mejoren su rendimiento, incurriendo en ocasiones en técnicas de dopaje. Actualmente, el dopaje es el resultado de una combinación de factores sociales, individuales, fisiológicos y culturales, que afecta no sólo a los deportistas profesionales, sino también a los deportistas aficionados. Para que su control y erradicación sea eficaz, es necesario un conocimiento del problema y de las sustancias más utilizadas, dentro de las cuales merecen especial atención las sustancias hormonales por su compleja detección y sus posibles repercusiones sobre la salud.

Palabras clave. Dopaje hormonal. Esteroides anabolizantes. Hormona de crecimiento. Eritropoyetina. Insulina.

\begin{abstract}
Sporting competition in our society has become the spectacle that mobilises and brings together the greatest number of people throughout the world, with the corresponding cultural and economic influence that this implies. As a result, the desire for athletic prowess has led sportspersons to undergo intense training programs and to consume substances that improve their performance, at times having recourse to doping techniques. At present, doping is the result of a combination of social, individual, physiological and cultural factors, which affect not only professional, but also amateur sportspeople. In order for the control and eradication of doping to be efficient, it is necessary to understand the problem and the substances that are most employed, amongst which special mention is merited by hormonal substances due to the complexity of detecting them and their possible repercussions on health.
\end{abstract}

Key words. Hormonal doping. Anabolic steroids. Growth hormone. Erythropoietin. Insulin.
1. Departamento de Farmacología de la Escuela Universitaria de Fisioterapia de Soria. Universidad de Valladolid. Soria.

2. Unidad de Endocrinología. Servicio de Medicina Interna. Complejo Hospitalario Santa Bárbara. Soria.

Aceptado para su publicación el 4 de noviembre de 2005.

\section{Correspondencia:}

Consuelo Laudo Pardos

Área de Farmacología

Escuela Universitaria de Fisioterapia de Soria

(Universidad de Valladolid)

C/ Nicolás Rabal, 17

42003 Soria

Tfno. 975129186

Fax: 975129 101/975 129102

E-mail: claudo@biocel.uva.es 


\section{INTRODUCCIÓN}

Desde hace unos años, en la practica deportiva y especialmente en el mundo de la alta competición, los deseos de superación atlética, empujados a menudo por las contraprestaciones sociales derivadas del éxito, así como los beneficios económicos, conducen al deportista a la utilización de los avances más recientes en su preparación y entrenamiento o ayudas ergogénicas, recurriendo también en numerosas ocasiones al uso de sustancias o métodos prohibidos, incurriendo así en el "dopaje".

En 1963, el Consejo de Europa adoptó la primera definición de dopaje como "la administración a una persona sana, o la utilización por ella misma de cualquier sustancia extraña al organismo o de sustancias fisiológicas, en cantidades o por vías anormales, con la intención de incrementar de una manera artificial o ilegal su rendimiento en competición". Posteriormente, ante la necesidad de establecer un concepto universal, la Comisión Médica del Comité Olímpico Internacional (COI) lo definió como "la administración de sustancias pertenecientes a grupos prohibidos de agentes farmacológicos y/o el empleo de diversos métodos prohibidos"'.

Recientemente, la Declaración de Lausana (1999), en la Conferencia Mundial sobre el dopaje en el deporte, lo definió como "el uso de un artificio (sustancia o método), potencialmente peligroso para la salud de los deportistas y/o susceptible de mejorar su rendimiento, o la presencia en el organismo de un deportista de una sustancia, o la constatación de un método, que figuren en la lista anexa al Código Antidopaje del Movimiento Olímpico"2.

Hoy día, el dopaje constituye el mayor problema que se extiende sobre la competición deportiva, dejando ésta de ser algo positivo, leal y solidario. La necesidad de su prevención, control y erradicación exigen una correcta educación de los valores éticos del deporte y una completa información de los posibles riesgos que pueden tener sobre la salud del deportista, con efectos secundarios a corto, medio o largo plazo, que son bien conocidos en algunos casos, pero en otros no tanto y que pueden llevar a poner en peligro la vida del deportista.

\section{ANTECEDENTES Y EVOLUCIÓN DEL DOPAJE}

Frente a la creencia de que el dopaje es un fenómeno reciente, existe documentación de prácticas relacionadas con él (pan empapado con opio, preparados a base de hierbas y setas) desde épocas remotas como la Grecia Clásica y la Roma Antigua, utilizadas especialmente para contrarrestar los efectos de la fatiga, prevenir las lesiones o estimular el espíritu de combate ${ }^{3-5}$, aunque, es a partir del siglo XIX cuando se tiene constancia de la utilización de sustancias de forma fraudulenta para mejorar el rendimiento deportivo. Así, el uso de estimulantes fue una práctica muy extendida durante la primera mitad del siglo pasado, que se vio desplazada en los años 70 por el dopaje hormonal, pues estas sustancias en ese momento resultaban difíciles de detectar ${ }^{5}$.

Ante el creciente consumo de sustancias, el COI ha ido poniendo en marcha a lo largo de los últimos años nuevas normas para luchar contra el dopaje, coordinando los esfuerzos de las diferentes organizaciones deportivas y de los poderes públicos. Así, la celebración en 1999 de la primera "Conferencia Mundial sobre el dopaje en el deporte", celebrada en Lausana por iniciativa del COI, marcó un cambio de rumbo en la forma de abordar dicho problema. Ese mismo año se acordó constituir y poner en funcionamiento la Agencia Mundial Antidopaje (AMA), cuya sede central se encuentra en Montreal. El Consejo de ésta fundación está integrado por representantes de organizaciones gubernamentales, intergubernamentales y deportivas, encontrándose España entre los países participantes.

En el año 2003, la AMA elaboró el "Código Mundial Antidopaje" con normas internacionales constituidas por un conjunto de reglas y directrices de obligado cumplimiento por las federaciones deportivas internacionales. De igual modo, se establecieron los criterios homologables del funcionamiento de laboratorios y la elaboración de una lista de sustancias y métodos prohibidos que fuera aceptada por el mayor número de países. Ese mismo año, el "Código Mundial Antidopaje" se aprobó por unanimidad 6 . Pero, el pasado mes de octubre de 2005, tuvo lugar su ratificación parla- 
mentaría, para darle validez jurídica, como "Convenio Internacional contra el dopaje en el deporte" en la UNESCO (Organización de Naciones Unidas para la Educación, la Ciencia y la Cultura). Actualmente, la lista de sustancias y métodos prohibidos, aprobada por la AMA y adoptada por la Comisión Médica del COI, no está cerrada, sino que se encuentra sometida a continuas modificaciones, incluyendo el término de "sustancias relacionadas", donde se incorporan los nuevos fármacos que van apareciendo en el mercado. Las últimas modificaciones han entrado en vigor a principios del 2006 $(\text { Tabla } 1)^{7}$.

Tabla 1. Sustancias y métodos prohibidos por la AMA?
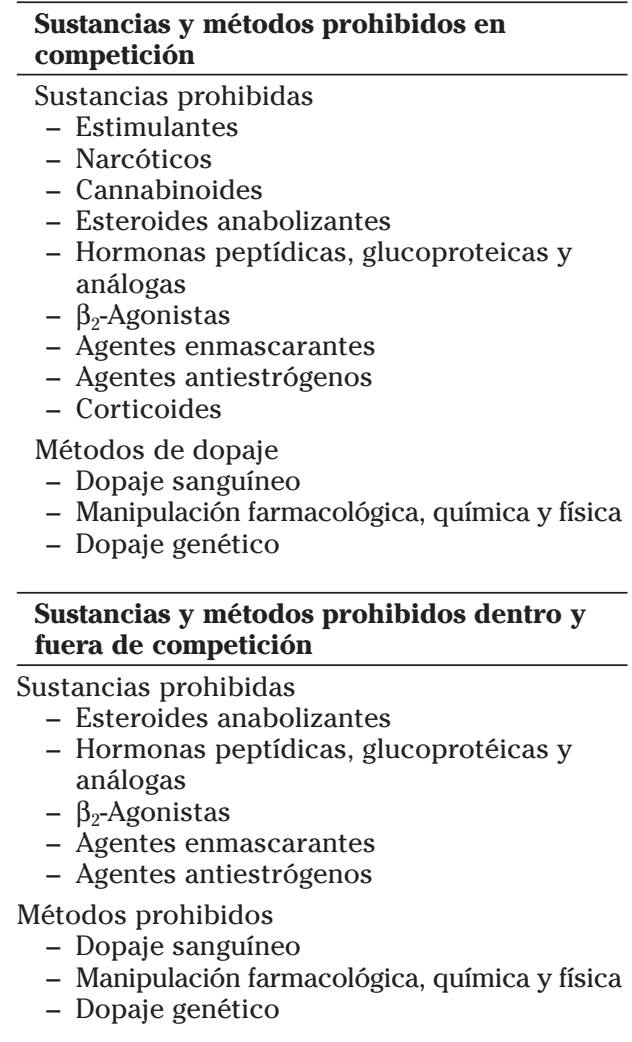
Sustancias prohibidas en deportes concretos
- Alcohol
- Beta-bloqueantes

Sustancias específicas
España, en materia de control antidopaje, adopta las directrices de la Agencia Mundial; su normativa se revisa periódicamente por el Consejo Superior de Deportes (CSD), recogiéndose las últimas modificaciones en la Resolución de 21 de diciembre de 2005, publicada en el Boletín Oficial del Estado (BOE) del 26 de diciembre de $2005^{8}$.

La presente revisión recoge la información sobre la prevalencia, la eficacia y los posibles efectos adversos del consumo de uno de los grupos de sustancias prohibidas más utilizadas en el ámbito deportivo, como son las sustancias hormonales.

\section{ESTEROIDES ANABOLIZANTES}

El término de esteroides anabolizantes (EA) hace referencia a un conjunto de compuestos relacionados con la testosterona tanto desde un punto de vista estructural como de su actividad biológica, aunque por su función asociada, deberían denominarse esteroides anabolicoandrogénicos ${ }^{9}$.

En el deporte comenzaron a utilizarse hacia los años cincuenta, siendo pioneros un equipo soviético de halterofilia ${ }^{4}$, para posteriormente extenderse su utilización fraudulenta a deportes de fuerza y/o de resistencia, con el fin de incrementar la masa muscular, la fuerza y la potencia. Incluso en ocasiones, con su consumo se pretende incrementar la agresividad en competición, fenómeno percibido por algunos atletas como algo positivo. Éste y otros efectos psicológicos se incrementan si coexiste una historia psiquiátrica, de abuso de alcohol u otras drogas ${ }^{10,11}$.

Se incluyeron en la lista de sustancias prohibidas por el COI en los juegos de verano de Montreal de 1976. Desde entonces se han sucedido numerosos escándalos deportivos por su utilización como por ejemplo en los Juegos Olímpicos de Seul (1988) la descalificación de Ben Johnson, el primer escándalo deportivo por consumo de estanozolol tras lograr el récord de los $100 \mathrm{~m}$ lisos. Recientemente ha arruinado el prestigio de figuras consagradas como Tim Montgomery, sancionado por el Tribunal de Arbitraje Deportivo (TAS) y desposeído de su marca. Actualmente, los EA están catalogados en la lista de la AMA, 
como sustancias prohibidas tanto dentro como fuera de la competición?

\section{Efectos fisiológicos}

La testosterona es la principal hormona masculina segregada por el testículo. Su síntesis, que oscila entre 5 y $10 \mathrm{mg}$ al día, se produce a partir del colesterol, bajo un mecanismo de retroalimentación negativo hipotálamo-hipofisario en respuesta a las gonadotropinas. Esta hormona también se produce, aunque en menor porcentaje, en la glándula suprarrenal y, en la mujer, también se sintetiza en dicha glándula y en el ovario, pero aquí en cantidades menores. Es responsable en el varón de la diferenciación sexual y del desarrollo de los caracteres sexuales secundarios masculinos, pero también actúa sobre el tejido muscular, óseo y hematopoyético ejerciendo un efecto anabolizante, incrementando la retención de nitrógeno, la masa magra corporal y el peso. Estas acciones parecen estar mediadas a través del mismo receptor androgénico, siendo el factor limitante para la acción de los esteroides anabolizantes el número de complejos hormona-receptor existente.

La administración oral de testosterona no es eficaz por su vida media corta, pues se metaboliza rápidamente en el hígado, pero su aislamiento ha facilitado el desarrollo de numerosos esteroides sintéticos. El objetivo de las modificaciones químicas en la molécula de testosterona es producir análogos activos con una absorción mantenida o enlentecida y con un metabolismo hepático disminuido, habiéndose obtenido andrógenos activos por vía oral (metiltestosterona, oxandrolona, estanozolol y oximetolona entre otros) y por vía parenteral (ésteres de testosterona como propionato, cipionato y enantato, ésteres de nandrolona y metenolona). Estos últimos, dada su lenta liberación a la circulación, requieren una administración menos frecuente y presentan menor toxicidad hepática que los esteroides orales ${ }^{12}$.

\section{Indicaciones médicas}

Su utilidad terapéutica es muy variada como hipogonadismo masculino, pubertad retrasada, estados de desnutrición en el síndrome de inmunodeficiencia adquirida
(SIDA), catabolismo inducido por corticoides, anemia grave, angioedema hereditario, rehabilitación postoperatoria y tratamiento de fracturas ${ }^{13}$. Estudios recientes también les atribuyen mejoras en lesiones musculares y capacidad de reducir la atrofia muscular propia de la inmovilización ${ }^{14,15}$. Incluso con dosis bajas, también parece reportar beneficios en las mujeres con fracturas de cadera, mejorando la densidad mineral ósea por inhibición de los osteoclastos $^{16,17}$. No se contempla ninguna otra indicación terapéutica, salvo para intentar aumentar de forma fraudulenta el rendimiento deportivo en los sujetos jóvenes y sanos, incrementando la masa muscular, la fuerza y la eritropoyesis.

\section{Aplicación en el deporte}

\section{Prevalencia}

Desde los años 60, los EA han sido una de las sustancias ergógenicas más utilizadas, desplazando a otras como los estimulantes. Los deportistas suelen referirse a ellos como el "desayuno de los campeones", lo que hace sospechar su amplia difusión y consumo. Durante años su uso parecía confinado a los levantadores de peso y culturistas pero, progresivamente, en los últimos años su empleo se ha ampliado a otras disciplinas.

Actualmente, se acepta que altas dosis de EA son capaces de incrementar el rendimiento deportivo, al aumentar la fuerza, la masa y la potencia muscular. Incluso puede modificar su actitud frente al entrenamiento, permitiéndole entrenarse con más frecuencia, tiempo e intensidad y con menor fatiga, aumentando así su competitividad $^{18}$.

\section{Formas de uso}

Para intentar obtener los mayores efectos anabolizantes, reduciendo su posibilidad de detección o evitar la aparición de tolerancia a un anabolizante en particular, se suelen emplear en ciclos de 6 a 12 semanas, de forma oral y/o intramuscular, para después suspenderlos durante un período más o menos prolongado o seguir consumiéndolos a una dosis más baja. En ocasiones, estas sustancias se utilizan con un "patrón piramidal", empezando con una 
dosis diaria baja al inicio del ciclo hasta alcanzar la dosis máxima, para luego ir disminuyéndola gradualmente hasta su retirada. También, es común utilizar otros fármacos para contrarrestar alguno de sus efectos secundarios o evitar su detección.

Las dosis utilizadas superan a las terapéuticas entre 10 y 100 veces, combinando con frecuencia varios preparados, orales y parenterales, denominando a dicha práctica "de hacinamiento" o "principio de cañonazo".

Actualmente, se están investigando nuevos métodos de detección, aunque sigue siendo en orina donde tiene lugar la mayoría de las determinaciones. Así en los últimos años han ganado popularidad aquellos andrógenos que son difíciles de detectar, o se metabolizan y excretan en pocos días, en particular el estanozolol y la testosterona. En los Juegos Olímpicos de Atenas, se detectó la tetrahidrogestrinona (THG), un nuevo esteroide sintético, hasta ahora indetectable en orina ${ }^{19}$. Todavía más reciente es el descubrimiento por la AMA, de otro anabolizante, la desoximetiltestosterona (DMT) (Tabla 2), (esteroi-

Tabla 2. Esteroides anabolizantes prohibido por la AMA?

Bolasterona
Boldenona
Clostebol
Danazol
Dehidroclorometiltestosterona
Desoximetiltestosterona
Estanozolol
Fluoximesterona
Mesterolona
Metandienona
Metenolona
Nandrolona
Noretandrolona
Oxandrolona
Oximesterona
Tetrahidrogestrinona (THG)
Testosterona
Otras sustancias con estructura química o
efectos biológicos similares

des anabolizantes prohibidos por la $\mathrm{AMA}^{\top}$ ). Pero el mayor reto lo constituye la detección del consumo de esteroides endógenos como la testosterona. Actualmente (2005) se ha sugerido que cuando la relación del cociente $\mathrm{T}$ (Testosterona)/EP (Epitestosterona) en orina es superior a cuatro, se podría considerar dicha muestra positiva, aunque antes de afirmarse debería investigarse si se trata de falsos positivos producidos por una situación patológica, déficit enzimático o ingesta de carne contaminada por EA. En caso de investigación se compararían con controles realizados con anterioridad. Si no se dispone de pruebas anteriores, el deportista será sometido a controles sin previo aviso, al menos, en tres ocasiones durante un período de 3 meses. Si el deportista rehusa a colaborar, se considerará la sustancia como dopante ${ }^{7}$.

\section{Efectos secundarios}

La información sobre los efectos adversos de los esteroides anabolizantes principalmente proceden de estudios observacionales $^{20,21}$ y aunque las manifestaciones menores son comunes (88-96\%), rara vez son letales. Suelen estar relacionados con el tipo de esteroide, dosis utilizada, frecuencia, edad, etc. La mayoría de los efectos son reversibles si se suspende su consumo, pero algunos son permanentes. Los más frecuentes incluyen acné (40-54\%), atrofia testicular (40-51\%), ginecomastia (10-34\%), estrías cutáneas (34\%) y dolor en el punto de inyección $(36 \%)^{18}$. Igualmente se han notificado graves alteraciones de la función hepática, tumores, peliosis hepática y angiosarcoma hepático especialmente con las administraciones por vía oral. También efectos cardiovasculares que pueden poner en riesgo la vida como hipertensión, miocardiopatía, arritmias y trombosis ${ }^{22,23}$. Diferenciando por sexo, las mujeres son muy sensibles a los anabolizantes, pudiendo aparecer virilización con amenorrea, hirsutismo, cambios en la voz, etc., alteraciones algunas irreversibles. En los varones, puede aparecer atrofia testicular, esterilidad y ginecomastia, y con menor frecuencia: hipertensión arterial, edema e hipertrofia prostática benigna. En el caso de los niños también se produciría dismi- 
nución de la talla definitiva por cierre epifisario precoz. También pueden provocar agresividad, irritabilidad, cambios de humor y efectos variables en la libido. En consumidores importantes se han descrito cuadros de depresión mayor o psicosis, incluso varios casos de dependencia física y suicidio ${ }^{11,18,24}$. Por otro lado, la inmunidad humoral también puede verse afectada, aunque su significado clínico es desconocido. Otros efectos adversos descritos son un mayor riesgo de lesiones musculotendinosas, al parecer por disminución en la elasticidad de los tendones, que con un aumento de la fuerza muscular predisponen a dichas lesiones en los atletas que los consumen ${ }^{13}$. Los anabolizantes orales también disminuyen las lipoproteínas de alta densidad (HDL), mientras que las lipoproteínas de baja densidad (LDL) permanecen sin cambios o aumentan.

\section{HORMONAS PEPTÍDICAS, GLUCOPROTEICAS Y ANÁLOGAS}

Las siguientes hormonas de naturaleza peptídica son sustancias que cuando exceden su rango normal en el deportista (se debe descartar que sean secundarias a situaciones fisiológicas o patológicas) están prohibidas, considerando que la muestra contiene una sustancia dopante. Igual sucede con sus miméticos (sustancia con efecto farmacológico similar a otra sustancia) y sus análogos (sustancia derivada de la modificación o alteración de la estructura química de otra sustancia y que produce un efecto farmacológico similar). Especialmente son utilizadas en deportes de fuerza, resistencia etc.

\section{Hormona de crecimiento}

La hormona de crecimiento (GH) es de naturaleza proteica, su síntesis se realiza en las células somatotropas de la adenohipófisis, con una producción diaria de 0,4-1 $\mathrm{mg}$ en los varones adultos y algo mayor en los adolescentes y las mujeres. Circula mayoritariamente unida a proteínas de transporte, con una vida media que oscila entre 17 y 45 minutos. Es liberada a la circulación de forma pulsátil, fundamentalmente bajo control de dos péptidos hipotalámicos (hormona liberadora de $\mathrm{GH}$ o
GhRH y somatostatina), aunque otros factores pueden influir en su liberación como el ejercicio (uno de los estímulos más potentes), el estrés y la hipoglucemia; también la ingestión de aminoácidos puede producir incrementos transitorios de $\mathrm{GH}^{25}$. Recientemente un nuevo péptido denominado "grelina", producido fundamentalmente en el estómago, está considerado como un potente liberador de $\mathrm{GH}^{26,27}$.

$\mathrm{El}$ incremento de los niveles de $\mathrm{GH}$ con el ejercicio son mayores cuanto más precoz y más intenso es el mismo; el pico se produce entre 15 y 20 minutos después de finalizarlo, alcanzando su máximo a los 4060 minutos y permaneciendo elevada varias horas. También influye el tipo de ejercicio que se realiza, si es o no intermitente (mayor respuesta) ${ }^{28}$ y el miembro que los realiza (mayor con los brazos que con las piernas) $)^{29}$.

\section{Efectos fisiológicos}

Durante la infancia, su principal papel fisiológico es promover el crecimiento somático a través de la producción, en el hígado, del factor de crecimiento insulínico I (IGF-I) o somatomedina C, que media numerosas acciones de la $\mathrm{GH}$, tales como la síntesis de DNA, RNA y proliferación celular, favoreciendo el crecimiento de tejidos esqueléticos y extraesqueléticos. También ejerce un papel central en el control del metabolismo proteico y en la composición corporal en los adultos ${ }^{30}$.

Tras el cierre epifisario también actúa sobre el metabolismo intermediario favoreciendo el anabolismo proteico y la lipólisis. Entre sus efectos metabólicos destacan: el incremento de la captación de aminoácidos y su incorporación a proteínas, especialmente en músculo e hígado y el incremento de la movilidad de ácidos grasos libres procedentes del tejido adiposo.

\section{Indicaciones médicas}

En la actualidad, existen diversas indicaciones aceptadas del tratamiento con GH en nuestro país, debiendo ser aprobados todos los casos por un Comité de Expertos. Se están estudiando otras posibles indicaciones futuras: ancianos, situaciones de estrés y úlceras ${ }^{31}$. 


\section{Aplicación en el deporte}

Es una sustancia dopante catalogada por la $\mathrm{AMA}^{7}$ como prohibida tanto dentro como fuera de la competición. Probablemente su consumo se ha incrementado entre la comunidad deportiva, tanto en profesionales como aficionados por su dificultad en la detección y se consume más especialmente desde que se dispone de GH sintética, aunque sin evidencias científicas de que mejore el rendimiento atlético, por lo menos a corto plazo.

Muchos deportistas consumidores creen que puede aportar los beneficios de los esteroides anabolizantes sin ninguno de sus efectos secundarios y sin poderse detectar; por otro lado, en niños dotados atléticamente, pero con talla normal-baja, puede resultar atrayente, para entrenadores y padres, la posibilidad de mejorar la talla y a la vez reducir los depósitos de grasa. Otro método de suplementación sería mediante la ingestión oral de fármacos capaces de estimular la secreción de GH endógena como propranolol, vasopresina, clonidina y L-dopa, o bien aminoácidos también estimulantes de la secreción de GH como arginina, lisina, ornitina y triptófano ${ }^{32}$.

Recientemente, con su consumo se han detectado incrementos en la síntesis proteica, reducción en la oxidación de las proteínas (que tiene lugar durante la realización de ejercicio físico) e incremento en la masa magra corporal ${ }^{33}$, aunque no parece mejorar el consumo de oxígeno ${ }^{34}$.

El mayor problema se plantea actualmente, por la dificultad en la detección del consumo de $\mathrm{GH}$, que tiende a resolverse basándose especialmente en técnicas por inmunoensayo dirigidas a diferenciar isoformas moleculares de $\mathrm{GH}$ recombinante ${ }^{35}$.

\section{Efectos adversos}

Los atletas suelen afirmar que la $\mathrm{GH}$ no tiene efectos secundarios, lo que probablemente sea debido a que emplean una forma inactiva (debe conservarse en frío), adulterada (con suero fisiológico, $\mathrm{GH}$ de primate) o una dosis incorrecta. Hasta el momento no se ha determinado la seguridad de la $\mathrm{GH}$ exógena en sujetos normales, aunque están bien definidos clínicamente los efectos de la hipersecreción endógena de GH.
El exceso de GH en los niños puede producir gigantismo por la aceleración del crecimiento óseo longitudinal. En adultos, la hipersecreción endógena prolongada de GH causa acromegalia que, entre otras, cursa con miopatía, neuropatía periférica, diabetes mellitus y enfermedad cardíaca, incluyendo cardiopatía isquémica, hipertensión arterial y miocardiopatía, por lo que sin tratamiento suelen fallecer antes de cumplir los 60 años. La enfermedad músculo-esquelética y cardíaca asociada con el exceso de $\mathrm{GH}$ puede ser irreversible, incluso normalizando los niveles de la hormona. Es posible que aquellos individuos expuestos a tratamientos prolongados de $\mathrm{GH}$ y con dosis extremadamente elevadas, puedan presentar un cuadro similar al de los pacientes acromegálicos. Hasta el momento, no hay casos de acromegalia descritos entre los deportistas que emplean la hormona, aunque se han comunicado signos precoces de la enfermedad ${ }^{36}$.

En pacientes con déficit de $\mathrm{GH}$ en la infancia, tratados con la hormona en edad adulta, se ha objetivado retención hídrica con aumento de peso y edema periférico; así como síndrome de túnel carpiano y artralgias. En algunos pacientes, el edema se resolvió de modo espontáneo, pero otros requirieron reducir la dosis. Teóricamente estos efectos adversos también podrían aparecer en deportistas que utilizan dosis mayores de $\mathrm{GH}^{19}$.

Otro problema potencial del empleo de la hormona exógena es el desarrollo de anticuerpos contra la GH que podrían interferir con la actividad de la hormona endógena y causar un déficit relativo de la misma. Finalmente, se deben tener en cuenta posibles problemas derivados de medidas higiénicas inadecuadas, pues se podría transmitir hepatitis, sida e incluso, con la de origen humano, la enfermedad de Creutzfeldt-Jakob.

\section{Eritropoyetina}

La eritropoyetina (EPO) es una hormona glucoproteica que se sintetiza principalmente en el riñón (90\%) y el resto en el hígado. En la actualidad también se encuentra disponible en cantidades ilimitadas al obtenerse mediante ingeniería genética ${ }^{37}$. 
El mayor estímulo para su producción lo constituye la hipoxemia, a partir de un sensor situado en el túbulo proximal del riñón, estimulando las células progenitoras de los eritrocitos, dando lugar a un aumento del hematocrito y la hemoglobi$\mathrm{na}^{38}$. Estos cambios aumentan el aporte de oxígeno a los tejidos, pudiendo mejorarse la capacidad para realizar ejercicio aeróbico mantenido, que depende del aporte y utilización del oxígeno a los músculos.

\section{Indicaciones médicas}

Su utilidad clínica la constituye la anemia producida por la insuficiencia renal terminal (IRT), aunque con posterioridad se ha utilizado con éxito en la anemia debida a otras causas, como la de la artritis reumatoide, la secundaria a procesos malignos, inducida por quimioterapia (especialmente con cisplatino), la anemia hemolítica, la aplasia de células rojas, la anemia de los prematuros e incluso la secundaria al tratamiento con zidovudina en el sida ${ }^{39}$.

La dosis y la frecuencia de administración determinan el incremento del hematocrito observado al administrar la hormona, siendo mejores los resultados cuando se administra de modo subcutáneo. A pesar de que las concentraciones de EPO regresan a la normalidad a la semana de la última dosis administrada, sus efectos se mantienen, dado que el incremento en la vida media de los hematíes que logra la EPO se mantiene durante 3 ó 4 meses después de haberla retirado. Es aconsejable, que la supresión del tratamiento con EPO se realice con disminuciones progresivas para evitar la aparición de neocitolisis ${ }^{40}$.

\section{Aplicación en el deporte}

La EPO, al incrementar el número de hematíes y la oxigenación muscular, es capaz de aumentar el rendimiento en ciertos deportes de resistencia, como el ciclismo profesional y el esquí de fondo ${ }^{41,42}$. Se cree que puede ayudar a reducir el esfuerzo fisiológico durante el ejercicio e incluso acelerar la recuperación tras el entrenamiento.

Recientemente, el consumo de esta sustancia está empezando a ser un problema no sólo entre los deportistas profesio- nales, sino también entre aficionados y jóvenes deportistas. Actualmente, con más frecuencia se recurre a la administración de eritropoyetina recombinante humana (rHuEpo), lo que ha permitido evitar el riesgo de infección y el inconveniente del almacenamiento que requiere el dopaje sanguíneo. Su administración ha demostrado ser eficaz para aumentar la concentración de hemoglobina (Hb), el consumo máximo de oxígeno $\left(\mathrm{VO}_{2} \mathrm{máx}\right)$ y la capacidad de trabajo físico ${ }^{41,43}$.

En la actualidad, su detección constituye un reto en el control del dopaje, ya que la EPO recombinante no se puede distinguir fácilmente de la hormona endógena. Por ello, la medición del hematocrito (Hct) es una forma indirecta, pero útil, de detectar el consumo de esta sustancia prohibida; así, un hematocrito superior al 50\% se considera demasiado elevado para la salud del deportista estableciéndolo como límite superior permitido. También se está procediendo a nuevos métodos de detección indirectos como a través de marcadores bioquímicos y sanguíneos de eritropoyesis alterada ${ }^{44,45}$ (Hct de reticulocitos, receptor de transferrina, \% de macrocitos etc.) mediante técnicas de ELISA y métodos fotométricos ${ }^{46}$; junto con técnicas de detección en orina ${ }^{47}$, por lo que se recurre a un doble sistema de control de sangre y orina ${ }^{48}$.

\section{Efectos adversos}

La mayoría de los efectos secundarios en los pacientes con IRT están en relación con un aumento de la viscosidad sanguínea secundaria a la elevación del hematocrito, incluyendo cuadros trombóticos, convulsiones e hipertensión. Estos efectos no se han documentado en los sujetos sanos, aunque existen informes que relacionan muertes inesperadas en varios ciclistas con el abuso de EPO.

Estos riesgos asociados al consumo de la hormona, pueden ser mayores en deportistas de resistencia, donde se pueden dar problemas de hemoconcentración debida a la presencia de deshidratación asociada al ejercicio prolongado o a los cambios de volumen plasmático posturalmente induci$\operatorname{dos}^{49}$. También pueden influir otros facto- 
res, como la presencia de una gran variabilidad interindividual en la respuesta al fármaco y la persistencia del estímulo en la médula ósea varios días después de la última administración, pudiendo seguir aumentando peligrosamente la viscosidad sanguínea.

Actualmente, para evitar problemas en las recogidas de muestras de sangre, se tiende a proceder a extracciones al principio de la mañana y no inmediatamente después del ejercicio, para evitar la posibilidad de elevaciones del Hct producidas por un cuadro de deshidratación aguda.

Probablemente, durante los próximos años se asista a la aparición de problemas relacionados con el dopaje sanguíneo como un incremento en la aparición de casos de sobrecarga de hierro, eritrocitosis de origen desconocido, anemias inexplicadas, complicaciones tromboembólicas atípicas, etc ${ }^{50}$.

\section{Insulina}

Es una hormona de naturaleza peptídica producida por las células beta pancreática. Considerada como una de las hormonas con acción anabólica más importante del organismo, su acción es decisiva para el transporte de nutrientes, como las proteínas o los hidratos de carbono. Actualmente, algunos deportistas, como los culturistas, la están utilizando como sustancia dopante, consumiéndola para disminuir el catabolismo a nivel muscular, promoviendo la síntesis de glucógeno y favoreciendo la entrada de glucosa en la célula muscular ${ }^{51}$.

Como sucede con otras sustancias, no es inocua y su consumo conlleva riesgo de hipoglucemias que pueden conducir a un coma y a ciertos trastornos sobre el metabolismo lipídico. En numerosas ocasiones, los deportistas, para evitar la aparición de hipoglucemias, recurren junto con la administración de insulina, al consumo de alimentos o bebidas con alto contenido glucídico.

La utilización de la insulina como sustancia dopante parece haberse extendido notablemente en el ámbito deportivo por ser un compuesto con una vida media muy corta, por lo que desaparece rápidamente del organismo, siendo difícil de detectar, incluso de diferenciarla de la del propio atleta. Recientemente se está procediendo a realizar controles de dopaje para análogos de insulina, mediante espectrofotometría de masa, considerado el sistema de detección más importante ${ }^{52}$.

Finalmente, su consumo esta prohibido en los deportistas, tanto dentro como fuera de la competición, excepto en atletas con diabetes mellitus insulino-dependiente, en cuyo caso, previamente a su consumo, lo certificará por escrito el equipo médico correspondiente o un especialista en endocrinología.

\section{Otras hormonas}

Los deportistas han recurrido también al consumo de otras sustancias como la gonadotropina coriónica (HCG), sustancia prohibida sólo en hombres y utilizada para estimular la secreción de testosterona, con supuestos efectos anabólicos sobre el tejido muscular, incrementando la masa muscular y esquelética. La corticotropina (ACTH) incrementaría la concentración de corticoides endógenos que podrían alterar el humor y el rendimiento máximo $^{53}$. La administración de $\mathrm{GnRH}$, secretada por el hipotálamo, regularía la producción y la secreción de las gonadotropinas. En el varón, la hormona luteinizante (LH) también está prohibida, ya que incrementaría la producción de testosterona, ejerciendo un efecto anabólico en el tejido muscular.

El consumo de estas sustancias hormonales en los deportistas, rara vez tiene lugar de forma aislada; generalmente, y en especial en algunas modalidades como los culturistas, es muy frecuente que recurran a la polifarmacia, donde asocian tres o más fármacos diferentes, incluyendo desde HCG, GH y hormonas tiroideas hasta estimulantes como cannabis, anfetaminas, cocaína, incluso sustancias enmascarantes.

Todo lo expuesto en la presente revisión, nos lleva a compartir los principios recogidos en la Carta Olímpica y señalados por el Presidente del COI, Jacques Rogge: "Un campeón es más que un ganador. Un campeón es alguien que respeta las reglas, rechaza el dopaje y compite dentro del espíritu del Juego Olímpico". 


\section{BIBLIOGRAFIA}

1. International Olympic Committee. International Olympic Committee Medical Code. Chapter II: prohibited classes of substances and prohibited methods. Lausanne, 31 de octubre de 1996.

2. Consejo Superior de Deportes (CSD). http://www.csd.mec.es/ Acceso 28/11/2005.

3. Thein LA, Thein JM, LANDRY GL. Ergogenic aids. Phys Ther 1995; 75: 426-439.

4. CATLIN DH, HatTon CK. Use and abuse of anabolic and other drugs for athletic enhancement. Adv Intern Med 1991; 36: 399-424.

5. Rodríguez Bueno C. "Historia del dopaje". En: Dopaje. Ed. Interamericana. Mc Graw-Hill. Madrid 1992: 7-17.

6. Casajus JA. Dopaje, salud y deporte. Inf Ter Sist Nac Salud 2005; 29: 1-11.

7. Agencia Mundial Antidopaje (AMA). http://www.wada-ama.org/ Acceso Enero de 2006.

8. Boletín Oficial del Estado (BOE $\mathrm{n}^{\mathrm{o}}$ 308), Lunes 26/12/2005.

9. WILSON JD. Androgen abuse by athletes. Endocr Rev 1988; 9: 181-199.

10. Brower KJ. Anabolic steroid abuse and dependence. Curr Psych Rep 2002; 4: 377387.

11. PoPe HG, Kouri EM, Hudson JI. Effects of supraphysiological doses of testosterona on mood and agresión in normal men: a randomized controlled trial. Arch Gen Psychiatry 2000; 57: 133-140.

12. Laudo C, Puigdevall V, Del Río MJ. Sustancias hormonales utilizadas como agentes ergogénicos. Med Clin 1999; 112: 67-73.

13. Simó CANonge R. Doping por esteroides anabolizantes. Endocrinología 1998; 45: 219-226.

14. Beiner JM, Jokl P, Cholewicki J, PANJABi MM. The effect of anabolic steroids and corticosteroids on healing of muscle contusion injury. Am J Sports Med 1999; 27: 2-9.

15. TAYLOR DC, BROOKS DE, RYAN JB. Anabolicandrogenic steroid administration causes hypertrophy of immobilized and non-immobilized skeletal muscle in a sedentary rabbit model. Am J Sports Med 1999; 27: 718-727.

16. Hedstrom M, Sjoberg K, Brosjo E, Astrom K, Sjoberg H, Dalen N. Positive effects of anabolic steroids, vitamin $\mathrm{D}$ and calcium on muscle mass, bone mineral density and clinical function after a hip fracture. A randomised study of 63 women. J Bone Joint Surg Br 2002; 84: 497-503.
17. Huber DM, Bendixen AC, Pathrose P, SRivastava S, Dienger KM, ShEvDE NK et al. Androgens suppress osteoclast formation induced by RANKL and macrophage-colony stimulating factor. Endocrinology 2001; 142: 3800-3808.

18. Evans NA. Current concepsts in anabolicandrogenic steroids. Am J Sports Med 2004; 32: 534-542.

19. ToKISH JM, Kocher MS, HaWKINS RJ. Ergogenic aids: A review of basic science, performance, side effects, and status in sports. Am J Sports Med 2004; 32: 1543-1533.

20. BOLDING G, SHERR L, ELFORD J. Use of anabolic steroids and associated health risks among gay men attending London gyms. Addiction 2002; 97: 195-201.

21. Evans NA. Gym \& tonic: a profile of 100 male steroid users. Br J Sports Med 1997; 31: 5458.

22. Kutscher EC, Lund BC, PerRy PJ. Anabolic steroids: a review for the clinician. Sports Med 2002; 32: 285-296.

23. Parssinen M, Seppala T. Steroid use and long term health risks in former athletes. Sports Med 2002; 32: 83-94.

24. HERNÁNDEZ M, GUILLEN JL. Abuso de anabolizantes. Psiq Biol 2005; 12: 18-21.

25. Puigdevall V, Laudo C, Del Río MJ, Velasco A. La hormona de crecimiento como agente ergogénico. Archiv Med Deporte 1996; 56: 447-451.

26. Kojima M, Hosoda H, Date Y, NaKazato M, MatSUO H, KangaWA K. Ghrelina is a growth-hormone-releasing acylated peptide from stomach. Nature 1999; 402: 656-660.

27. Soriano Guillén L, Barrios V, ARgente J. Implicaciones clínico-diagnósticas del péptido ghrelina. An Pediatr 2004; 60 (Supl. 12): 30-35.

28. Raynaud J, Capderou A, Martineau JP, BorDACHAR J, DURAND J. "Intersubject variability in growth hormone time course during different types of work". J Appl Physiol 1983; 55: 1682-1687.

29. Kozlowski S, Chwalbinska-Moneta J, Vigas M, Kaciuba-Uscilko H, NazaR K. Greater serum $\mathrm{GH}$ response to arm than to leg exercise performed at equivalent oxygen uptake. Eur $\mathrm{J}$ Appl Physiol 1983; 52: 132-135.

30. Wallace JD, Cuneo RC. Growth hormone abuse in athletes: A Review. Endocrinologist 2000; 10: 175-184.

31. Ministerio de Sanidad y Consumo. Comunicación sobre riesgos de medicamentos para profesionales sanitarios (Riesgos del uso de hormona de crecimiento en personas sanas y paso a "uso hospitalario"). "Agencia espa- 
ñola de medicamentos y productos sanitarios". Ref: 2005/08; 22/4/2005.

32. RENNIE MJ. Claims for the anabolic effects of growth hormone: a case of the Emperor's new clothes? Br J Sports Med 2003; 37: 100-105.

33. Healy ML, Gibney J, Russell-Jones DL, Pentecost C, Croos P, SönKsen H et al. High dose growth hormone exerts an anabolic effect at rest and during exercise in endurancetrained athletes. J Clin Endocrinol Metab 2003; 88: 5221-5226.

34. Berggren A, Ehrnborg C, Rosen T, Elleggard L, Bengtsson BA, CaIDahl K. "Short-term administration of supraphysiological recombinant human growth hormone (GH) does not increase maximum endurance exercise capacity in healthy, active young men and women with normal GH-insulin-like growth factor I axes. J Clin Endocrinol Metab 2005; 90: 3268-3273.

35. Bidlingmaier M, Wu Z, Strasburger CJ. Test method: Baillieres Best Pract Res Clin Endocrinol Metab 2000; 14: 99-109.

36. EHRnBorg C, Bengtsson B, Rosén T. Growth hormone abuse. Baillieres Best Pract Clin Endocrinol Metab 2000; 14: 71-77.

37. FlahaRTy KK, GRIMM AM, VlasSes PH. Epetin: himan recombinant erythropoietin. Clin Pharm 1989; 8: 769-782.

38. MoRENO M. Tratamiento con eritropoyetina de la anemia asociada a la infección por el virus de la inmunodeficiencia humana. Med Clin (Barc) 1977; 108: 266-271.

39. Cazzola M, Mercuriali F, Brugnara C. Use of recombinant human erythropoietin outside the setting of uremia. Blood 1997; 89: 42484267.

40. Mateos JM. Eritropoyetina. Pasado y algunas preguntas futuras. Nefrología 1999; XIX (Supl. 3): 1-4.

41. EKBlom B, Berglund B. Effect of erythropoietin administration on maximal aerobic power. Scand J Med Sci Sports 1991; 1: 88-93.

42. Birkeland KI, Stray-Gundersen J, HemmERSABACH P, Hallen J, HaUg E, BaHr R. Effect of rhEPO administration on serum levels of sTfR and cycling performance. Med Sci Sports Exerc 2000; 32: 1238-1243.
43. Audran M, Gareau R, Matecki S, Durand F, Chenard C, Sicart MT et al. Effects of erythropoietin administration in training athletes and possible indirect detection in doping control. Med Sci Sports Exerc 1999; 31: 639-645.

44. Lacombe C, MayeuX P. Biology of erythropoietin. Haematologica. 1998; 83: 724-732.

45. BREYMANN C. Erythropoietin test methods. Baillieres Best Pract Res Clin Endocrinol Metab 2000; 14: 135-145.

46. Parisotto R, Gore CJ, Emslie KR, Ashenden MJ, Brugnara C, Howe $\mathrm{C}$ et al. Un nuevo método que utiliza marcadores de eritropoyesis alterada para la detección de abuso de eritropoyetina recombinante humana en deportistas. Archiv Med Deporte 2000; 79: 421-432.

47. Gareau R, Audran M, Baynes RD, Flowers CH, Duvallet A, Senecal L et al. Erythropoietin abuse in athletes. Nature (Lond) 1996; 380: 113.

48. Pascual JA, Belalcazar V, De Bolos C, GutiérREZ R, Llop E, Segura J. "Recombinant erythropoietin and analogues. A Challenge for doping control". Ther Drug Monit 2004; 26 : 175-179.

49. SIMON TL. Induced erythrocytemia and athletic performance. Semin Hematol 1994; 31: 128-133.

50. Cazzola M. Una estrategia global para la prevención y la detección de dopaje sanguíneo con eritropoyetina y drogas relacionadas. Archiv Med Deporte. 2000; 79: 413417.

51. Evans PJ, LYNCH RM. Insulin as a drug of abuse in body building. $\mathrm{Br} \mathrm{J}$ Sports Med 2003; 37: 356-357.

52. Thevis M, Thomas A, Delahaut P, Bosselor A, SCHANZER W. "Qualitative determination of synthetic analogues of insulin in human plasma by immunoaffinity purification and liquid chromatography-tandem mass spectrometry for doping control purposes". Anal Chem 2005; 77: 3579-3585.

53. Soetens E, De Meirleir K, Hueting JE. No influence of ACTH on maximal performance. Psychopharmacology (Berl) 1995; 118: 260-266. 
